

Rudolf Kammerl

30.7.2001

\section{Ethische Aspekte heteronomer und autonomer Moral netzbasierter} Kommunikation

Internetbasierte Kommunikation wird zunehmend Bestandteil der Mediensozialisation Jugendlicher. In Hinblick auf die beliebte Freizeitaktivität «Chatten», werden Zusammenhänge zwischen struktureller Bedingungen internetbasierter Kommunikation und Formen heteronomer wie autonomer Moral dargestellt und aus medienethischer Perspektive problematisiert.

\section{Medienethische Dimensionen internetbasierter Kommunikation}

Durch die zunehmende Integration des Internet als festen Bestandteil medialer Alltagswelt für einen rasch wachsenden Teil der Bevölkerung, insbesondere für die 14-19-jährigen ${ }^{1}$ und deren mediale Freizeitgestaltung, rücken die aus medienethischer Sicht problematischen Aspekte netzbasierter Kommunikations- und Informationspraxis als dringliche Handlungsbedarfe in den Vordergrund. Vor dem Hintergrund eines auf die abendländische Tradition christlicher-humanistischer Überlieferungen bezugnehmenden Wertesystems und ohne die vielfältigen Formen prosozialen Engagements vieler Internetnutzer ausser Acht lassen zu wollen, können viele Phänomene des internetbasierten Medienalltags als nicht moralische beschrieben werden. Bizarrste Formen von Sexualität und Gewalt, radikalste Formen politischer Meinung werden dargeboten, vereinzelt werden sogar Frauen, Kinder und Organe im Internet gehandelt. Häufig wird gelogen, geflamed ${ }^{2}$, gespamed ${ }^{3}$,

${ }^{1}$ Neuere GfK-Studien weisen über 60\% dieser Altersgruppe als Internetnutzer aus (z. B. Online-Monitor 7. Untersuchungswelle). Damit sind die Jugendlichen die Altergruppe mit dem höchsten Anteil an Internetnutzern.

2 Flaming meint das Auftreten unerwarteter stark emotionalisierter personenbezogener Kommentare (Flames) - meist in Form von Beleidigungen, Wutausbrüchen o.ä., aber auch in Form von Liebeserklärungen und Sympathiebekundungen. Flames werden $1 / 17$
Hacker- und Virenattacken richten grossen Schaden an, wirtschaftliche und staatliche Organisationen durchleuchten die Privatsphäre der Nutzer und Datenbanken von Organisationen im grossen Stil (Cookies, Echelon). Weitere relevante Themen sind Zensur und Ausschluss bestimmter (Welt)bevölkerungsgruppen - die Liste aus medienethischer Perspektive zu thematisierende Problemfelder könnte sicherlich noch fortgesetzt werden ${ }^{4}$. Mit der hier getroffenen Auswahl wird bereits deutlich, dass sowohl Individuen, Gruppen, Organisationen, Regierungen bezogen auf das Internet sowohl als Täter wie auch als Opfer in Erscheinung treten können. Ethische Aspekte des Internet müssen sich deshalb auf Mikro-, Meso- und Makroebene der Nutzung dieses Mediums beziehen. Sie betreffen nicht nur die Moral einzelner Internetnutzer, sondern auch Normen in Gruppen und Organisationen, aber auch rechtliche Aspekte und die Frage nach übergeordneten, möglicherweise universalen ethischen Prinzipien.

In diesem Beitrag steht die Freizeitkommunikation zwischen Einzelnen sowie in Gruppen im Mittelpunkt. Insbesondere Chats und daraus resultierende E-Mail-Sequenzen wurden daraufhin untersucht, welche moralischen Prinzipien durch die strukturellen Bedingungen dieser Formen der internetbasierten Kommunikation als handlungsanleitende Orientierungshilfen unterstützt oder erschwert werden. Dabei interessiert vor allem die Frage, wie die Möglichkeit einzuschätzen ist, dass sich in diesen Kommunikationsfeldern verallgemeinerbare moralische Prinzipien als selbstgesetzte Handlungsmaximen der Kommunikationspartner etablieren können. Diese Frage ist relevant für die individuelle Moralentwicklung, sowie für die allgemeine Entwicklung einer Kommunikationskultur für den virtuellen Kommunikationsraum.

Um auf diese Fragen Antwort geben zu können, gehe ich in folgenden Schritten vor: Zunächst werden ausgehend von der übergeordneten internetethischen Grenzbereichen einige Strukturbedingungen der internetbasierten Kommunikation skizziert und in einem zweiten Schritt daraufhin befragt, inwiefern sie welche moralischen Orientierungen begünstigen oder verhindern. Im letzten Teil meines Beitrags gehe ich der Frage nach, wie

mit dem Fehlen von Konventionen bei der internetbasierter Kommunikation, sowie mit den mangelnden Hinweisen über die Kommunikationspartner geklärt, was häufig zu Überattribuierung und Stereotypenbildung führt.

3 Spammen ist das Versenden von unerwünschten Werbebotschaften an Mailinglisten und Newsgruppen.

${ }_{4}$ Viele der genannten Problemfelder sind keine internetspezifischen. Auch im konventionellen Telefonnetz bspw. wird gelogen, betrogen und vieles andere mehr. 
allgemeine medienethische Massstäbe für die netzbasierte Kommunikation Geltung beanspruchen können.

Eine erste grobe Eingrenzung meiner Betrachtungen haben bereits vorliegende Arbeiten zu einer Interethik vorgegeben, insbesondere die Beiträge von Sandbothe (z. B. 1999), Capurro (z. B. 2000) und Debatin (z. B. 1999). Im Anschluss an Debatin (1999) können vor allem Wissen, Freiheit und Identität als allgemeine Grenzbereiche einer Internetethik herausgestellt werden.

Wissen/Information ${ }^{5}$ : Das Internet bietet eine unübersichtliche, rasch anwachsende Menge von Informationen unterschiedlichster Qualität und Thematik an. Eine Orientierung bedarf in dieser anschwellenden Informationsflut der Selektion. Ausgehend vom Wissen um die Möglichkeit der Informationsmanipulation wird die Überprüfbarkeit der Informationen hinsichtlich qualitativer und ethischer Kriterien zu einem entscheidenden Bewertungsmassstab für dieses Medium. Bezogen auf die netzbasierte Kommunikation im Freizeitbereich betrifft dieser Aspekt sowohl die Aussagen der Internetnutzer sowie die Informationen über sie.

Freiheit: Die vielfältigen und häufig genutzten Interaktions- und Kommunikationsmöglichkeiten im Internet stellen einen neuen öffentlichen Raum dar. Für diesen neuartigen Raum muss die Frage nach Graden und Arten von Regulierungen und Freiräumen erst noch bestimmt werden. Hier sind Möglichkeiten und Konsequenzen von Fremdregulierung und Selbstkontrolle (des Einzelnen wie auch der Netzgemeinschaft) abzuwägen.

Identität konstituiert sich durch Selbstwahrnehmung und Fremdwahrnehmung in Auseinandersetzung mit der Umwelt. Damit wird auch internetbasierter Kommunikation zugebilligt, Relevanz für die Identitätsbildung haben zu können. Das Potential des Internet wird hierbei sehr unterschiedlich eingeschätzt. So finden sich im Rahmen der philosophischen Anthropologie z. B. die Befürchtung, dass durch Virtualität des Kommunikationsraums Internet Körperlichkeit auf ein nutzlos gewordenes Anhängsel («Wetware», «Restmüll des Fleischlichen») reduziert wird. Die derzeit

${ }^{5}$ Es scheint mir nicht angebracht, wie z. B. Debatin 1999, das Internet als «gigantisches gesellschaftliches Gedächtnis» oder als «universalen Wissensspeicher» zu bezeichnen. Medien «wissen» nichts. Wissen ist an natürliche Intelligenz, d.h. an menschliches Bewusstsein gebunden. Erst durch die Verarbeitung durch ein menschliches Bewusstsein wird Information zu Wissen (vgl. Pollak/Kammerl 2000, 237f). vorliegenden Befunde aus der empirischen Medienforschung ${ }^{6}$ zeichnen aber ein differenzierteres Bild und weisen auf sehr unterschiedliche Muster der individuellen Aneignung netzbasierter Kommunikation hin. Mit dem hier ausgewählten Aspekt «Moral in der Internetkommunikation» wurde versucht, einen für Fremd- und Selbstwahrnehmung sehr relevanten Bereich zu erfassen.

\section{Anonymität im virtuellen Raum begünstigt unmoralisches Verhalten}

Im Bereich der internetbasierten Freizeitaktivitäten erfreut sich die Kommunikation mittels Chat, Mail und News grosser Beliebtheit. Der Austausch über unterschiedlichste Themen mit völlig unbekannten Internetnutzern, sowie das gegenseitige Kennenlernen stellt für viele einen besonderen Reiz dar. Längst sind das internetbasierte Schwätzchen, die elektronische Brieffreundschaft oder der themenspezifische Austausch über die schwarzen Bretter des virtuellen Raums zu einem Massenphänomen geworden. In dem Masse in dem die Kommunikation via Internet sich als Phänomen des Medienalltags verbreitet, vermehren sich auch die Berichte der Internetnutzer über mehr oder weniger starke Verstösse gegen einfache Konventionen zwischenmenschlicher Kommunikation. Hierbei handelt es sich um eine grosse Bandbreite möglicher Erscheinungsformen (vgl. z. B. Reed-Steere 2000). Sie reichen von kleineren oder grösseren Unhöflichkeiten, - die eher als Frage der Etikette abgetan werden könnten, - über Lügen, Beleidigungen, gezielten Irreführungen bis hin zu harten verbalen Attacken (z. B. im Sinne eines sexuellen Missbrauchs) und informatischer Attacken (z. B. flooding, mailbombs etc.), die auf der Grundlage in unserer Gesellschaft geltender Moralvorstellungen nicht nur als unmoralisch bewertet werden können, sondern darüber hinaus zum Teil auch gegen geltendes Recht verstossen. Auf der Grundlage alltäglicher Konventionen der Kommunikation als «Fehlverhalten» und /oder «unmoralisch» zu bewertendes Verhalten wird vor allem durch die Anonymität und das weitgehende Fehlen von nachhaltigen Sanktionsmöglichkeiten bei Normverstössen begünstigt. Der Kommunikationspartner, der mir in den Chaträumen oder in den Newsforen begegnet, ist unbekannt. Sofern es sich nicht um einen Videochat handelt, sind nonverbale Informationen über die Person ausgeblendet. Informationen über den Gegenüber, die zur Verfügung stehen, sind hoch selektiert und manipuliert. Bei der Kommunikation via Internet bleibt es darüber hinaus im Dunkeln, wer sich hinter dem Nickname, hinter einer

\footnotetext{
${ }^{6}$ Z. B. Döring 1999; Thiedeke 2000; Thimm 2000
} 
E-Mailadresse und hinter der präsentierten virtuellen Identität wirklich verbirgt. Es bleibt zunächst offen, wie authentisch der Kommunikationspartner ist. Hinter einer virtuellen Identität kann sich jemand völlig anders verbergen. Denkbar (und empirisch vorfindbar), dass mir ein und derselbe Mensch mit verschiedenen virtuellen Identitäten zeitgleich oder zeitversetzt im Internet begegnet. Die Möglichkeiten, seine Anonymität zu wahren, sind sehr vielfältig und schränken die Sanktionsmöglichkeiten sehr ein. Jemand, der zu einem Chat nicht mehr zugelassen, oder als Kommunikationspartner nicht mehr akzeptiert werden würde, kann einfach eine andere Identität annehmen um diese Sanktionen zu umgehen. Die wenigen Spuren, die er hinterlässt, reichen meistens nicht, um einen Internetnutzer zu identifizieren. Die Ahndung von Internetkriminalität stösst hier häufig auf ihre Grenzen. Noch stärker begrenzt ist aber die Möglichkeit der Ächtung unmoralischen, aber nicht straffälligen Verhaltens. Der Kommunikationsraum Internet ist durch die machbare Anonymität der Internetnutzer in weiten Bereichen der Kommunikation in News, Chats und daran anschliessenden E-Mailsequenzen weitgehend frei von Sanktionsmöglichkeiten bei Normverstössen.

Es wäre interessant zu wissen, wie Norbert Elias diese Facette des Alltags internetbasierter Kommunikation kommentiert hätte. Im Sinne seiner Zivilisationstheorie (Elias 1976) könnten viele der Phänomene internetbasierter Kommunikation - wie z. B. die starke Dominanz sexueller Inhalte oder die sich in Flames oftmals ausdrückende impulsive Aggressivität einiger Internetnutzer auf das Fehlen eines Gewaltmonopols in diesem Kommunikationsraum (das durch die Anonymität der Internetnutzer und der damit weitgehend garantierten Sanktionsfreiheit de facto zur Zeit existiert) sowie auf eine nur wenig ausgebildete Selbstkontrolle zurückgeführt werden. Affekte und Triebe dienen als Verhaltensorientierungen, wenn keine externen oder verinnerlichten Verhaltenskontrollen diese kontrollieren. Elias hat die zunehmende Verinnerlichung ehemals extern kontrollierter Verhaltensanforderungen als einen historischen Prozess skizziert. Moral wäre im Sinne Elias Resultat der Reproduktion von Verhaltensstandards durch externen und internen Zwang. Das Bürgertum der Aufklärung grenzte sich in seinem Selbstverständnis von der blossen äusseren Zivilisiertheit der Adligen durch diese Verinnerlichung ab. Internalisierte Normen zeigen sich vor allem in den Verhaltensorientierungen in sanktionsfreien Räumen. Hierbei ist auch an Bereiche zu denken, in denen die Sanktionierung gewisser Verhaltensanforderungen (noch) nicht gewährleistet ist - wie eben z. B. bei den genannten Konstellationen internetbasierter Kommunikation. Am (kommuni- kativen) Handeln des Einzelnen zeigt sich, inwiefern er extern sanktionierte Verhaltensstandards verinnerlicht hat oder ob hier Affekte handlungsanleitend sind.

Ebenso lassen diese Verhaltensorientierungen Aufschlüsse über die Dominanz heteronomer bzw. autonomer Formen von Moral zu. Die Entwicklung autonomer, moralischer Verhaltensdispositionen wird als Bestandteil von Bildungsprozessen und als Ausdruck einer geglückten Identitätsbildung angesehen. Medienethische Appelle, vor allem aber die Bemühungen um Moralerziehung bzw. Herstellung moralischer Urteilskompetenz konzentrieren sich auf eine dauerhafte Veränderung von Verhaltensdispositionen. Weniger als die Vermittlung bestimmter vorgegebener Verhaltensweisen steht hier das Ideal der autonom moralisch entscheidenden Persönlichkeit im Vordergrund. Auf der Grundlage sich selbst gesetzter moralischer Prinzipien soll das eigene Verhalten selbst reflektiert und reguliert werden.

Die allgemeine Bedeutung moralischer Dispositionen sollte aber freilich nicht zu hoch eingeschätzt werden. Situationsspezifische Variablen haben gegenüber den dispositionalen Variablen eine grössere Aussagekraft, wenn es um die Erklärung, Vorhersage und Kontrolle von Verhalten geht (Krumm 1999, 309ff). In diesem Sinne wäre ein Rückschluss von unmoralischem Verhalten auf das Fehlen adäquater moralischer Dispositionen bzw. auf mangelhafte Moralerziehung nicht zulässig. Ein derartiges Wirkgefüge zu unterstellen, hiesse nicht nur die Möglichkeiten einer Moralerziehung bzw. moralischen Bildung zu überschätzen, sondern auch die Freiheit und Wechselhaftigkeit des menschlichen Willens zu unterschätzen.

Anonymität und Sanktionsfreiheit begünstigen unmoralisches Verhalten, sie können es aber nicht ausreichend erklären. Wie die Beispiele funktionierender harmonischer virtueller Gruppen und Gemeinschaften zeigen, sind sie keineswegs hinreichende Bedingungen unmoralischen Verhaltens, andererseits aber - wie aus dem Alltag der Unmoral bekannt ist - auch keine notwendigen Voraussetzungen des Regel- und Normverstosses. Als weiterer Faktor ist sicherlich die durch die Anonymität begünstigte Distanz zu nennen, die aber auch in der internetbasierten Kommunikation vor allem durch Bindung überwunden werden kann und dann meist auch zur Aufhebung der Anonymität führt ${ }^{7}$.

7 Das Aufeinandertreffen von Anonymität und Intimität, das Debatin als typische ChatKonstellation beschreibt, konstituiert noch nicht Bindung. 
Heteronome und autonome Moral in der netzbasierten Kommunikation

Im Anschluss an Piaget und Kohlberg kann die gelingende moralische Entwicklung als Übergang von einer heteronomen zu einer autonomen Moral beschrieben werden. Im Stadium der Heteronomie werden Regeln in ihrer Gültigkeit und ihrer Verhandelbarkeit nicht hinterfragt. Ihr Ursprung geht auf Autoritäten zurück, die auch berechtigt sind, Normabweichungen zu bestrafen. Im Stadium der Autonomie entscheiden Heranwachsende selbst, was gut und richtig ist und nehmen Einfluss auf die Normgestaltung. Piaget (1986) spricht erst dann von moralischer Autonomie, «wenn die gegenseitige Achtung stark genug ist, im Individuum das innerliche Bedürfnis hervorzurufen, den anderen so zu behandeln, wie es selbst behandelt sein möchte». Autonome Moral im Sinne Piagets ist nicht durch einseitigen Respekt vor moralischen Regeln und deren Sanktionierbarkeit sondern durch wechselseitigen Respekt von Personen gekennzeichnet. Bei Kohlberg folgt dieser Wechselseitigkeit in den weiteren Stufen postkonventioneller Moral eine zunehmende Orientierung an übergeordneten moralischen Prinzipien (vgl. z. B. Colby /Kohlberg 1986). Moralische Autonomie kann als freiwillige Selbstbindung einer Person an Überzeugungen, Ideale, Werte verstanden werden. Erst auf der Basis gewollter Selbstbindung kann wertend $\mathrm{zu}$ spontanen Impulsen und Umweltereignissen Stellung genommen werden. In dieser Selbstbindung wird somit auch Identität zum Ausdruck gebracht. Gelungene Ich-Identität beinhaltet konsistenten, kohärenten und kontinuierlichen Ausdruck dieser Selbstbindung in Verhalten und Stellungnahmen und macht Individualität identifizierbar.

Im Anschluss an die Studien zur moralischen Entwicklung lassen sich einige Strukturbedingungen von Kommunikation benennen, die eine signifikante Bedeutung für die Entwicklung autonomer Moral haben. Allgemein können stabile emotionale Zuwendung, soziale Anerkennung, Chancen zu Teilnahme an Kommunikationsprozessen, Möglichkeiten zur Mitwirkung an kooperativen Entscheidungsprozessen, Informationen über Folgen des eigenen Handelns, Gestaltungsmöglichkeiten als günstige Sozialisationsbedingungen, hingegen Geringschätzung, Indifferenz oder Unberechenbarkeit in der Wertschätzung, latent schwelende Konflikte, eingeschränkte Kommunikationsmöglichkeiten, überzogene Restriktion, sowie unklare, bzw. inadäquate Verantwortungszuschreibungen als ungünstige Bedingungen für die moralische Sozialisation von Jugendlichen ausgewiesen werden (vgl. Döbert/
Nunner-Winkler 1983; Oser 1986; Corsten /Lempert 1992).

Unter Berücksichtigung der Tatsache, dass für immer mehr Menschen die Kommunikation im Internet Bestandteil der medialen Sozialisation wird, gilt es zu untersuchen, welche dieser Strukturbedingungen hier als typische auffindbar sind und inwiefern sie Formen heteronomer und autonomer Moral begünstigen. Insbesondere die Frage nach dem Vorhandensein von Regelwerken sowie deren Verhandelbarkeit und Sanktionierung ist hier von engerem Interesse.

In diesem Beitrag möchte ich mich mit dieser Fragestellung auf die Chats konzentrieren. Chatten (plaudern, schwätzen) ist eine synchrone Kommunikationsform. Die Gesprächspartner schreiben sich mehr oder weniger zeitgleich Texte, die in nahezu Echtzeit auf dem Bildschirm des Gegenüber zu sehen sind. Der Schreibstil ähnelt dem der mündlichen Konversation sehr (vgl. Runkehl / Schlobinski / Siever 1998, 73ff). Es lassen sich vier verschiedene Chattypen unterscheiden.

Der Internet-Relay-Chat (IRC) ist der älteste und bislang am häufigsten verwendete Chattyp. Er ist textbasiert, erfordert ein spezielles Anwendungsprogramm (z. B. mIRC) und arbeitet unabhängig vom WWW.

Die Teilnahme am webbasierten Chat erfordert lediglich einen Java-fähigen Browser. Das Erscheinungsbild ähnelt dem IRC sehr.

Der Visual Virtual Chat zeichnet sich durch eine graphische Chat-Umgebung aus, die zum Teil von den Teilnehmern mitgestaltet werden kann. Die dazu notwendige spezielle Software kann aus dem Internet geladen werden. Der Videochat erfordert Webcams, mit denen die Chatter eine Videokonferenz aufbauen. Abhängig von der Übertragungsrate der Internetverbindung können dann die ansonsten nonverbalen Informationen live vermittelt werden. Wegen den technischen Voraussetzungen hat der Videochat bei den Internetnutzern noch nicht den Verbreitungsgrad wie der textbasierte Chat.

Soweit in den Chats Konversationsregeln explizit ausgewiesen werden (z. B. in Netiquette, Chatiquette, FAQs, o.a. ), sind sie meist fest vorgegeben und insbesondere für neuere Kommunikationspartner kaum verhandelbar. Ihre

${ }^{8}$ In institutionalisierten Kontexten pädagogischen Handelns hat das Wissen um die Bedeutung der Strukturen kommunikativen Handelns für die Entwicklung moralischer Urteilskompetenz sowie für moralisches Handeln zur Anwendung unterschiedlicher Konzepte geführt. Exemplarisch sei an dieser Stelle auf die JustCommunity-School (Kohlberg), die Kommunikative Didaktik (Schäfer/ Schaller) oder die realistischen Diskurse (Oser) verwiesen. 
Einhaltung wird vom Operator und/oder dem Moderator überwacht. Operator und Moderator sind Beauftragte des kommerziellen, institutionellen Anbieters oder werden von den Veteranen eines Channels gestellt. Normverstösse können u.a. zum Ausschluss aus dem Kommunikationskontext führen.

Döring / Schestag (2000) fassen auf der Grundlage einer Auswertung der Regelwerke von den textbasierten IRC-Channels vier übergeordnete Regelbereiche zusammen:

Keinen rechtswidrigen Aktivitäten mit Hilfe des IRC-Dienstes nachgehen (z. B. Rassismus, Sexismus, Antisemitismus, Nazi-Parolen, illegale Pornos ....) Keine unnötige Netzlast im IRC-Netzwerk erzeugen und IRC-Server nicht destabilisieren (keine kommerzielle Werbung, keine häufige Mode- oder Topic-Wechsel, keine Warskripts ....)

Keine Störungen der Channel-Kommunikation herbeiführen oder zulassen (keine unleserlichen, unnötigen oder überauffälligen Textströme, keine Unterminierung der Gruppenhierarchie, keine kollektiven technischen Angriffe (takeover) oder Gefährdungen, keine Verbreitung unangenehmer Stimmung - z. B. Fäkalsprache o.ä.)

Keine Beeinträchtigung einzelner Chatnutzer verursachen (keine technischen Angriffe, keine verbalen Angriffe, keine Nick-Fakes)

Die Einhaltung dieser Regel wird durch verschiedene Methoden der positiven und negativen Sanktionierung sichergestellt. Sowohl technische wie soziale Methoden kommen hier zum Zuge. Tabelle 1 zeigt die in IRCChannels von Döring und Schelstag vorgefundenen Sanktionierungsmöglichkeiten. $\mathrm{Zu}$ berücksichtigen ist, dass zwischen den verschiedenen Chattypen, aber auch zwischen den Channels die angewandten technischen Sanktionierungsmöglichkeiten variieren.

\begin{tabular}{|l|l|l|}
\cline { 2 - 3 } \multicolumn{1}{c|}{} & Technische Sanktionierung & Soziale Sanktionierung \\
\hline Sasitive & OP-Vergabe & Zuwendung \\
Sanktionierung & Bot-Master-Vergabe & Anerkennung \\
\hline Negative & Channel-Ban & Ignorieren \\
Sanktionierung & Channel-Kick & Spott \\
& $\begin{array}{l}\text { Op- oder Bot-Masterentzug } \\
\text { Streichung aus Toplist }\end{array}$ & $\begin{array}{l}\text { Zurechtweisung } \\
\text { Beschimpfung } \\
\text { etc. }\end{array}$ \\
\hline
\end{tabular}

Tab 1: Sanktionierungsmethoden in Internet Relay Chat-Channels (Döring/ Schestag 2000, S. 340)
Bei den Sanktionen im Chat handelt es sich um die Vergabe und den Entzug von Status, Macht und Partizipationsmöglichkeiten. Positive und negative technische Sanktionen werden - abgesehen von der Möglichkeit, Beiträge von bestimmten Teilnehmern durch den Befehl /IGNORE auszublenden von den Operators vergeben. Dies betrifft sowohl die Vergabe der Operatorund der Bot-Masterfunktion", (soweit vorhanden auch) die Streichung aus Toplists $^{10}$, sowie auch die Möglichkeit, einen anderen Internetnutzer temporär oder dauerhaft ${ }^{11}$ aus dem Channel auszuschliessen. Die Mittel der sozialen Sanktionierung können auch von den übrigen Chattern angewandt werden und sind in anderen sozialen Kontexten ausserhalb der internetbasierten Kommunikation ebenfalls auffindbar.

In den 12 von Döring und Schestag ausgewerteten Channels fanden in erster Linie Kicks und Kick-Bans Anwendung, um Verstösse gegen die Regelwerke zu sanktionieren. Innerhalb einer Stunde wurden - bei sehr ungleicher Verteilung über die Channels - 61mal Personen aus dem Kommunikationskanal temporär oder auf Dauer ausgeschlossen. Neben früherem Fehlverhalten waren hier vor allem Äusserungen mit sexuellem Bezug und das flooding ausschlaggebend, daneben waren aber auch persönliche Streitereien häufig die Ursache oder der Anlass der Sanktionierung blieb unklar. Angesichts der Härte der Sanktion - Ausschluss aus Kommunikation - stellt sich schon der Eindruck ein, dass zum Teil eine überzogen restriktive Sanktionierung vorliegt. Dieser Eindruck verstärkt sich durch die Beobachtung, dass nicht alle Normverstösse sanktioniert werden, sondern dass einige ungeahndet bleiben, da sie den Kontrollinstanzen entgehen.

Auch wenn die Diskursivität der Regeln zum Teil im Regelwerk behauptet wird, sieht die Anwendung keine Diskussion vor. Zum Teil ist die Bestrafung der Normverstösse automatisiert, ansonsten ist sie alleinige Entscheidung des Operators.

9 Die Bots (Robots) sind eigenständig agierende Programme, die im IRC-Netz in erster Linie eingesetzt werden, um zu verhindern, dass ein Channel einer feindlichen Übernahme zum Opfer fällt. Darüber hinaus erfüllen sie regulative und Dienstleistungsfunktionen.

${ }^{10}$ In einigen Channels wird in einer Liste der Top Talker eine Rangreihe der Stammgäste dokumentiert. Regelverstöße können dann durch Zurückstufung oder Streichung aus dieser Liste bestraft werden.

${ }^{11}$ Die Dauerhaftigkeit des Ausschlusses wird allerdings durch die unter den gegebenen technischen Möglichkeiten dadurch relativiert, dass dem so sanktionierten Internetnutzer die Option offen bleibt, unter einer anderen Identität neu Zugang zum Channel zu erlangen! 
Die Kommunikationsmöglichkeiten im Chat können als eingeschränkte bezeichnet werden. Insbesondere die Möglichkeit, internetbasierte kommunikative Prozesse selbst $\mathrm{zu}$ thematisieren und hierüber mitzuentscheiden ist sehr begrenzt. Ein solches Anliegen wird von Teilnehmern, die an einem thematischen oder hedonistisch orientierten Austausch interessiert sind, sehr schnell als störend abgetan. Anonymität, Distanz und die eingeschränkte nonverbale Kommunikation lassen die Internetnutzer lange über die tatsächlich gegenseitige Wertschätzung im unklaren.

Unter Beachtung dieser Strukturmerkmale ist der mediale Sozialisationsraum Chat m. E. nicht unbedingt zu den Freizeitangeboten für Kinder und Jugendliche zu zählen, die einer Förderung bedürfen. Auch wenn unter Beachtung einfacher Vorsichtsmassnahmen nicht von grösseren Gefährdungen ausgegangen werden muss, sind die hier dominierenden Kommunikationsstrukturen und die dadurch begünstigten Umgangsformen schon problematisch. Auch eine aktuelle stichprobenartige Überprüfung der von Gieger (2000) für Kinder und Jugendliche empfohlenen Chats (wie z. B. 〈www.rap.de〉) bestätigt mich in dieser tendenziellen Aussage.

Natürlich muss auch auf die andere Seite hingewiesen werden: Im Chat können sich auch ganz gelungene Sozialbeziehungen entwickeln. Eine starke subjektive Relevanz der Kommunikation mit anderen Internetnutzern ist Voraussetzung dafür, dass sich stabile Beziehungen herausbilden können, in denen Wertschätzung und Anerkennung erfahrbar wird. Virtuelle Kontakte können soziale Gratifikationen bieten und neue Gestaltungsräume eröffnen. Empathie und Bindung ${ }^{12}$ im Einzel- oder Gruppenkontakt führen zu prosozialen Orientierungen aus denen auch soziale Beziehung ausserhalb des Netzes entstehen können.

\section{Virtuelle Identität - virtuelle Moral?}

Für die Frage nach der autonomen, individuellen Orientierung an etwaigen individuellen moralischen Maximen ist die Bedeutung des Handelns im Internet für das Selbstkonzept entscheidend. Wann haben die Kommunikationspartner und deren Rückmeldungen hohe Bedeutung für die Sicht von sich selbst? In diesem Zusammenhang stellt sich die Frage, wann es wirklich gerechtfertigt ist, von virtuellen Identitäten zu sprechen. Der in der Literatur vorfindbare Begriff «Virtuelle Identität» unterstellt, dass Internetnutzer ver-

\footnotetext{
${ }^{12}$ Zur Stärkung der Bindung im Internet ist es wichtig Beziehungskiller zu vermeiden, Beziehungen zu intensivieren und Herzlichkeit/Wärme auszudrücken. $11 / 17$
}

schiedene Identitäten haben, neben der realen mindestens noch eine virtuelle (Real-Life-Identity vs. Virtual Identity), möglicherweise aber auch mehrere. In der Identitätstheorie wird heute durchaus die Ausbildung mehrerer Identitäten bzw. Teil-Identitäten nicht mehr als pathologische Erscheinungsform, sondern als Befreiung vom beengenden Identitätszwang betrachtet. Im Zeichen von Pluralisierung und fortschreitender gesellschaftlicher Differenzierung sei die »Fleckerlteppich-Identität« (Keupp 1997) Ausdruck zeitgemässer Identitätsbildung. In diesem Sinne wäre die virtuelle Identität eine von vielen, die mit den anderen Teil-Identitäten vermittelt über individuelle Konstruktionen nur noch wenige Anknüpfungspunkte oder gar keine mehr hat. Für das Selbstkonzept der Internetnutzer kann netzbasierte Kommunikation sowohl funktional im Sinne experimenteller Identitätsarbeit sein, aber auch Realitätsflucht (bis hin zur Internet Addiction Disorder), Spiel oder authentische Selbstdarstellung sein. Das Phänomen «virtuelle Identität» kann zwar im Rahmen postmoderner Identitätskonzepte (multiple Identität, Patchworkidentität z. B. bei Bilden 1997, Gergen 1996, Keupp 1997) - als mehr oder weniger entkoppelte Teilidentität interpretiert werden, aber auch als mehr oder weniger bedeutsames Kommunikationsfeld einer Gesamtidentität. ${ }^{13}$ Es wäre m. E. falsch jedem, der via Internet kommuniziert, eine virtuelle Identität zuzuschreiben. Von virtueller Identität kann nur dann die Rede sein, wenn die internetbasierte Kommunikation vom Internetnutzer hohe Bedeutung für das Selbstkonzept, das Selbstwertgefühl und für seine Kontrollüberzeugung zugeschrieben wird. Bei geringer Identitätsrelevanz netzbasierter Kommunikation ist bei negativen sozialen Sanktionen oder mangelnden Gratifikationen mit Abbruch der Kommunikation, mit Wechseln der inszenierten Figuren und bewussten Normverstössen zu rechnen. Eine Orientierung an bereichsübergreifenden individuellen moralischen Überzeugungen, die sich in Empathie und prosozialem Engagement ausdrückt, ist insbesondere dann $\mathrm{zu}$ erwarten, wenn es sich bei der netzbasierten Kommunikation um eine authentische ${ }^{14}$ und stabile handelt, mit hoher Identitätsrelevanz für alle Beteiligten. Je stärker das Interesse einer Person an der Kommunikationsteilnahme an Gruppenmitgliedschaft desto empfänglicher ist es auch für Sanktionierungsmassnahmen. Die optimistische Einstellung über eine allgemeine Sanktionierbarkeit von Döring / Schestag (2000, 344ff) kann ich aber nicht teilen, da provozierte Sanktionen (Kick,

\footnotetext{
${ }^{13}$ Das individuelle Bemühen um Konsistenz, Kohärenz und Kontinuität ist hierbei zu beachten. Vgl. hierzu ausführlich Fink/Kammerl 2001, 12ff.

${ }^{14}$ Einige Internetnutzer geben sogar an, im Netz authentischer zu sein als ausserhalb. 
Bann) den Initiator nicht wirklich treffen. Unmoralisches Verhalten im Netz kann also teilweise darauf zurückgeführt werden, dass zwischen Internetnutzern keine Bindung besteht und dass subjektives Handeln im virtuellen Raum nicht als moralisch bedeutsames Handlungsfeld bewertet wird.

\section{Welche normativen Bezugsrahmen können im virtuellen Raum Geltung beanspruchen?}

Medienethik betreibt ethische Reflexion der Handlungsnormen im Bereich der medienvermittelten Kommunikation und Information. Soweit hierbei auf das Internet bezug genommen wird, ist auch von Informationsethik, Cyberethik oder Internetethik die Rede (und beinhaltet sowohl netzbasierte Kommunikation und Information, sowie auch netzexterne auf das Internet bezogene Verhaltensformen und Urteile $)^{15}$. Ethik ist die Reflexionstheorie der Moral. Sie versucht moralische Urteile und geforderte Verhaltensweisen, die für einen Lebensbereich gültig sind, rational $\mathrm{zu}$ begründen und auf verallgemeinerbare moralische Prinzipien zurückzuführen (vgl. Funiok/ Schmälzle 1999, 19f.). Medienethik geht also über eine neutrale Beschreibung vorfindbarer Moral und Handlungsregeln hinaus und trägt eigene normative Bewertungsmassstäbe heran.

Wie kann der normative Bezugsrahmen begründet werden kann? Bezüglich der Frage nach einer allgemeinen Legitimierbarkeit normativer Aussagen herrschen unterschiedliche Positionen vor.

Normative Legitimation: Von obersten religiösen oder politischen Grundüberzeugungen, denen Gültigkeit oder Wahrheit unterstellt werden, werden Normen abgeleitet und deren Sanktionierung legitimiert. Die obersten Ausgangsnormen werden hierbei nicht weiter hinterfragt und stellen das weltanschauliche Fundament dar, aus denen sich die deduzierten Verhaltenserwartungen begründen.

$\mathrm{Ob}$ (und wie) ein (welcher) normativer Bezugsrahmen für das Internet bzw. im Internet Geltung beanspruchen und erlangen kann ist derzeit noch fraglich. Die Grenzen interkultureller Verständigung, der Wertepluralismus und Relativismus in westlichen Industrienationen scheinen normative Legiti-

\footnotetext{
${ }^{15}$ Wie ausserhalb des Netzes gibt es auch im Virtual Life moralisch agierende Subjekte und unmoralische. Es wäre trivial, wenn sich die Bemühungen einer Internetethik nur auf die in anderen Bereichen alltäglichen Handelns bereits untersuchten allgemeinen personeninternen und -externen Bedingungen beziehen könnte. Internetspezifische Merkmale der Kommunikationsstruktur begünstigen jedoch zum Teil durchaus internetspezifische Formen von Moral und Selbstkonzepte.
}

mationsmöglichkeiten, aber auch die Möglichkeiten, mittels anerkannter Verfahren kulturübergreifende Normen zu bestimmen, stark zu beschränken.

Bei der Verfahrenslegitimation wird auf formale, mehrheitlich anerkannte Entscheidungsverfahren zurückgegriffen. Die in diesen Verfahren gewonnenen Ergebnisse gelten als legitimiert, da sie Resultate rechtmässig beauftragter Institutionen sind, die innerhalb des sozialen Systems Anerkennung erfahren. Die Anerkennung ist in erster Linie formal, nicht inhaltlich begründet.

Im Sinne diskursiver Legitimation soll die Gültigkeit von Normen in herrschaftsfreien Diskussionen durch Konsens erreicht werden, die sich nur von der Kraft des besseren Arguments (statt von der Kunst der Überredung) leiten lassen. Anzustreben sei eine ideale, symmetrische Sprechsituation, in der alle Beteiligten einbezogen sind. In diesem Sinne wäre es Aufgabe von Medienethik, die kommunikativen Strukturen medialer Kommunikation zu hinterfragen. Die Fähigkeit diese Strukturen einer kritischen Reflexion zu unterziehen wäre auch Bestandteil einer auf die Medien bezogenen kommunikative Kompetenz, wie sie Baacke (1980) entwickelt hat.

Hier ist auch auf die Versuche hinzuweisen, die kulturelle Unabhängigkeit des Virtuellen Raums zu behaupten. Spätestens mit Barlows Proklamation («Declaration of the Independence of Cyberspace» 1996) wird dem Cyberspace das Existenzrecht als ein eigenständiger Kulturraum eingefordert. Das Einfordern externer Moralvorstellung käme einer Kolonialisierung der Internetkultur gleich. Statt dessen sei - so die Befürworter der Unabhängigkeit - auf die Selbstregulierung innerhalb der Netzkultur zu setzen. Die Kommunikationskultur des Internet kann nicht als eine einheitliche beschrieben werden. In dem weltweiten Medium Internet sind sehr heterogene Subkulturen mit kontextspezifischen Konventionen aufzufinden. In der Chat-Kommunikation mit zunächst unbekannten Netzteilnehmern überwiegen emotionale Orientierungen. Spielerische Aspekte, irrationale Zuschreibungen und Stereotypisierung, sowie daraus resultierende emotionale Überreaktionen (Flames) prägen den Verlauf virtueller Kontakte und die Entwicklung daraus entstehender Beziehungen sehr. Statt rational begründeter moralischer Prinzipien bestimmen emotionale Befindlichkeiten die Moral des individuellen Handelns ${ }^{16}$. Soweit man den Gedanken eines sich

${ }^{16}$ Schiller veranlasst Kants rigorose Ablehnung empathischer Neigungen als Motiv von Moral zu der Erwiderung: «Gern dien' ich den Freunden, doch tu ich es leider mit Neigung, und so wurmt es mich oft, dass ich nicht tugendhaft bin.» 
selbst regulierenden autonomen Kulturraums aufgreifen will, müsste man also ausgehend von der Chat-Kommunikation darauf hinweisen, dass die soziogenetische Moralentwicklung in dieser Kultur noch nicht so weit fortgeschritten ist.

Wichtiger aber scheint mir der an dieser Stelle nochmals anzubringende Hinweis, dass es sich bei den Mitglieder des Cyberspace eben nicht um die fremden Bewohner eines isolierten Kulturraumes handelt, sondern um Menschen aus den Industrienationen, die in ihrer Internetnutzung ihrer alltäglichen Erfahrungen, Ängste, Wünsche Befindlichkeiten etc. einbringen. Da der Nachweis, dass die multiple Persönlichkeit in unserer Gesellschaft statistischer Normalfall wäre, noch nicht erbracht ist, müssen Zusammenhänge zwischen der Moral der Internetnutzer im Netz und ausserhalb davon angenommen werden. Es ist zu erwarten, dass sich medienethische Maximen bei den Internetnutzern als Bestandteil handlungsanleitender Individualethiken nur dann etablieren können, soweit es innerhalb und ausserhalb des Mediums gelingt, mittels diskursiver Verfahren hierfür Verbindlichkeit einzufordern. Es ist zu erwarten, dass dies nur im Kontext einer allgemeinen moralischen Bildung gelingen kann.

\section{Literatur}

Baacke, D.: Kommunikation und Kompetenz. München/Weinheim 1980

Barlow, J.: «Cyberspace Independence Declaration.» 〈http://info.bris.ac.uk/ lwmdcg/DoI.html〉 1996 (10.06.2001)

Bilden, H.: «Das Individuum - ein dynamisches System vielfältiger Teilselbste.» In: Keupp, H./ Höfer, R. (Hrsg.): Identitätsarbeit heute. Frankfurt a. M. 1997

Capurro, R.: «Das Internet und die Grenzen der Ethik.» In: Rath, M. (Hrsg.): Medienethik und Medienwirkungsforschung. Wiesbaden 2000

Colby, A. / Kohlberg, L.: «Das moralische Urteil: der kognitionszentrierte entwicklungspsychologische Ansatz.» In: Betram, H. (Hrsg.): Gesellschaftlicher Zwang und moralische Autonomie. Frankfurt a. M. 1986.

Corsten, M./ Lempert, W.: Moralische Dimensionen der Arbeitssphäre. Berlin 1992

Debatin, B.: «Analyse einer öffentlichen Gruppenkonversation im ChatRoom.»〈http://www.uni-leipzig.de/ debatin/German/Chat.htm〉 1997 $(05.02 .2001)$ ders.: «Ethik und Internet. Zur normativen Problematik von OnlineKommunikation.» In: Funiok, R. / Schmälzle, U./ Werth, C. (Hrsg.): Medienethik - die Frage der Verantwortung. Bonn 1999

Döbert, R, / Nummer-Winkler, G.: Adoleszenzkrise und Identitätsbildung. Frankfurt a. M. $1982^{3}$

Döring, N.: Sozialpsychologie des Internet. Göttingen u.a. 1999

Döring, N./ Schestag, A.: «Soziale Normen in virtuellen Gruppen. Eine empirische Untersuchung am Beispiel ausgewählter Chat-Channels.» In: Thiedeke, U. (Hrsg.): Virtuelle Gruppen. Wiesbaden 2000

Elias, N.: Über den Prozess der Zivilisation. 2 Bde. Frankfurt a. M. 1976

Fink, G. / Kammerl, R.: «Virtuelle Identitäten als Ausdruck zeitgemässer Identitätsarbeit?» In: medien praktisch 01/2001.

Funiok, R.: «Stichwort Medienethik.»In: Hüther, Schorb, Brehm-Kloth (Hrsg.): Grundbegriffe Medienpädagogik. München 1997.

Funiok, R. /Schmälzle, U.: «Medienethik vor neuen Herausforderungen.» In: Funiok, R. / Schmälzle, U. / Werth, C. (Hrsg.): Medienethik - die Frage der Verantwortung. Bonn 1999

Gergen, K.: Das übersättigte Selbst. Heidelberg 1996

GfK Medienforschung: «Online-Monitor 7. Untersuchungswelle» 〈http://www.gfk.de/produkte/eigene_pdf/online_monitor.pdf $>2001$ (10.06.2001)

Gieber, C.: «Chatrooms für Kinder - Recherche und Analyse der Angebote.» http://www.dji.de/www-kinderseiten/chats.htm> 2000 (13.11.2000)

Keupp, H.: «Diskursarena Identität.» In: Keupp, H. / Höfer, R. (Hrsg.): Identitätsarbeit heute. Frankfurt a. M. 1997

Krumm, V.: «Bedingungen von Verhalten und pädagogisches Handeln.» In: Pollak, G. / Prim, R. (Hrsg.): Erziehungswissenschaft und Pädagogik zwischen kritischer Reflexion und Dienstleistung. Weinheim 1999

Marotzki, W.: «Zukunftsdimensionen von Bildung im neuen öffentlichen Raum.»In: Marotzki, W. /Meister, D. /Sander, U. (Hrsg.): Zum Bildungswert des Internet. Opladen 2000

Mikos, L.: «Erinnerung, Populärkultur und Lebensentwurf.» In: medien praktisch 01/1999 〈ttp://www.gep.de/medienpraktisch/amedienp/mp199/1-99miko.htm>. 1999 (05.02.01.)

Müller, K.: «Verdoppelte Realität - Virtuelle Realität? Erkenntnistheoretische, sozialphilospohische und anthropologische Konsequenzen der Neuen Medien.» In: Funiok, R. / Schmälzle, U. / Werth, C. (Hrsg.): Medienethik - die Frage der Verantwortung. Bonn 1999 
Oser, F. (Hrsg.): Transformation und Entwicklung. Frankfurt a. M. 1986

Piaget, J.: Das moralische Urteil beim Kinde. München 1986

Pollak, G. / Kammerl, R.: ««To know or not to know»: erziehungswissenschaftliche Bemerkungen zur Wissensgesellschaft.» In: Kammerl, R.: Computerunterstütztes Lernen. München, Wien 2000

Reed-Steere, E.: «Das Selbst und das Internet: Wandlungen der Illusion vom einen Selbst.» In: Thiedeke, U. (Hrsg.): Virtuelle Gruppen. Wiesbaden 2000

Runkehl, J. / Schlobinski, P. / Siever, T.: Sprache und Kommunikation im Internet. Opladen, Wiesbaden 1998

Sandbothe, M.: «Das Internet als Massenmedium: Neue Anforderungen an Medienethik und Medienkompetenz.»

〈http://www.uni-jena.de/ms/massmed.htm> 1999. (05.02.2001.)

ders.: «Wie der Ausflug in die Welt der virtuellen Quasselbuden unser reales Dasein bereichern kann.»〈http://www.uni-jena.de/ms/diewelt.htm〉2000. (05.02.2001.)

Thiedeke, U. (Hrsg.): Virtuelle Gruppen. Wiesbaden 2000.

Thimm, C. (Hrsg.): Soziales im Netz. Sprache, Beziehungen und Kommunikationskulturen im Internet. Opladen, Wiesbaden 2000

Turkle, S. Leben im Netz. Reinbek bei Hamburg 1998

Vogelgesang, W.: «Ich bin, wen ich spiele. Ludische Identitäten im Netz.» In: Thimm, C. (Hrsg.): Soziales im Netz. Opladen, Wiesbaden 2000 